\title{
Myocardial Motion Estimation in Tagged MR Sequences by Using $\alpha$ MI-Based Non Rigid Registration
}

\author{
E. Oubel ${ }^{1}$, C. Tobon-Gomez ${ }^{1}$, A.O. Hero ${ }^{2}$, and A.F. Frangi ${ }^{1}$ \\ 1 Computational Imaging Laboratory, Pompeu Fabra University, Barcelona, Spain \\ \{estanislao.oubel, catalina.tobon, alejandro.frangi\}@upf.edu \\ 2 Dept. of EECS, The University of Michigan, Ann Arbor (MI), 48109-2122, USA \\ hero@umich.edu
}

\begin{abstract}
Tagged Magnetic Resonance Imaging (MRI) is currently the reference MR modality for myocardial motion and strain analysis. NMIbased non rigid registration has proven to be an accurate method to retrieve cardiac deformation fields. The use of $\alpha \mathrm{MI}$ permits higher dimensional features to be implemented in myocardial deformation estimation through image registration. This paper demonstrates that this is feasible with a set of Haar wavelet features of high dimension. While we do not demonstrate performance improvement for this set of features, there is no significant degradation as compared to implementing the registration method with the traditional NMI metric. We use Entropic Spanning Graphs (ESGs) to estimate the $\alpha \mathrm{MI}$ of the wavelet feature vectors WFVs since this is not possible with histograms. To the best of our knowledge, this is the first time that ESGs are used for non rigid registration.
\end{abstract}

\section{Introduction}

Tagged magnetic resonance imaging (MRI) is a well established technique used to obtain regional information about the deformation of the left ventricle (LV) [1] , and thus potentially help to diagnose cardiovascular diseases. Basically, this technique consists in perturbating the magnetization of the myocardium in a specified spatial pattern at end-diastole. These perturbations appear as dark stripes or grids when imaged immediately after application of the tag pattern. Since the myocardium retains knowledge of this disturbance, the dark grids experience the same deformation the heart does as it contracts, allowing local strain parameters to be estimated.

Several methods have been proposed to retrieve LV deformation field: optical flow 34, Harmonic Phase (HARP) MRI [56], tag detection and tracking [7,8,9,10] and image registration [11,12]. The use of registration to estimate cardiac motion has proven to overcome many drawbacks existent on previous approaches.

The use of $\alpha \mathrm{MI}$ permits higher dimensional features to be implemented in myocardial deformation estimation and registration problems. In this paper, 
we aim to evaluate the performance of $\alpha \mathrm{MI}$ based registration methods with respect to gold standard measurements and with respect to NMI based image registration. Specifically, we use Haar wavelet coefficients at each pixel as feature vectors $(\mathrm{FVs})$ and ESGs to estimate the $\alpha \mathrm{MI}$ of these vectors.

This paper is organized in six sections. Section 2 explains how to estimate cardiac deformation fields by using image registration. In this section, the concept of $\alpha \mathrm{MI}$ and its estimation by using ESGs is also presented. Section 3 describes the dataset used for the experiments. Results are presented in Section 4 and discussed in Section 5 Finally, the conclusions can be found in Section 6.

\section{Method}

The registration algorithm we used, is based on the method originally developed by Rueckert et al. 13. for detection of cancerous lesions in contrast enhanced MR breast images. We modified this algorithm by replacing NMI with $\alpha \mathrm{MI}$ computed from Wavelet Feature Vectors (WFVs). The main problem derived from using vectors instead of intensity values, is that the curse of dimensionality forbids the use of histograms for probability density function (pdf) estimation. Therefore, in order to compute the $\alpha \mathrm{MI}$ of these vectors, we used $\mathrm{kNN}$ graph estimators which completely bypass pdf estimation [14.

\subsection{Motion Estimation}

To track cardiac motion throughout multiple time frames we used Multilevel Free Form Deformations (MFFDs) as suggested by Schnabel et al. [15, where the transformation $\mathbf{T}(\mathbf{u}, t)$ is represented as the sum of a series of local FFDs:

$$
\mathbf{T}(\mathbf{u}, t)=\sum_{p=1}^{t} \mathbf{T}_{\text {local }}^{p}(\mathbf{u}, t)
$$

Thus, the motion estimation starts registering the first two frames of the sequence $I(\mathbf{x}, 0)$ and $I(\mathbf{x}, 1)$, and a single FFD is obtained. Then, for the next frame $I(\mathbf{x}, 2)$, a new FFD is added and the frame is registered to $I(\mathbf{x}, 0)$ taking as initial transformation the one obtained for $I(\mathbf{x}, 1)$. This process is repeated for the remaining frames $I(\mathbf{x}, t)$ in the cardiac cycle. Once all the frames are registered to the first one, the MFFD consists of $N$ FFDs that model the myocardium deformation.

\subsection{Similarity Measure}

To recover the deformation field at time $t$, the image $I(\mathbf{x}, t)$ is registered to $I(\mathbf{x}, 0)$ by optimizing some cost function. Let $I_{s}$ and $I_{t}$ be random variables representing the source and target image, with pdfs $p_{s}\left(I_{s}\right)$ and $p_{t}\left(I_{t}\right)$ respectively. Let $p_{s t}\left(I_{s}, I_{t}\right)$ represent the joint pdf of $I_{s}$ and $I_{t}$. The $\alpha$ mutual information $(\alpha \mathrm{MI})$ of $I_{s}$ and $I_{t}$ is defined as: 


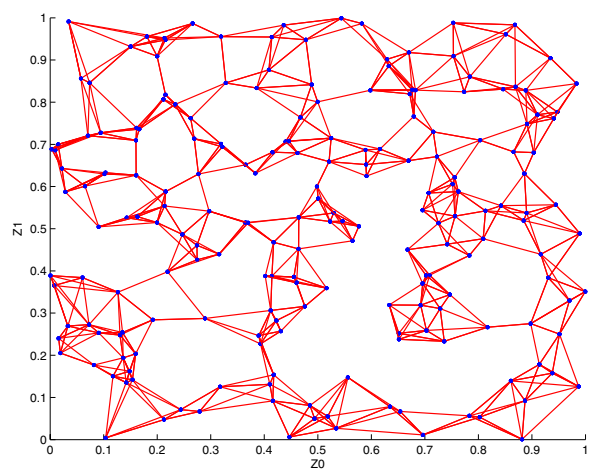

(a)

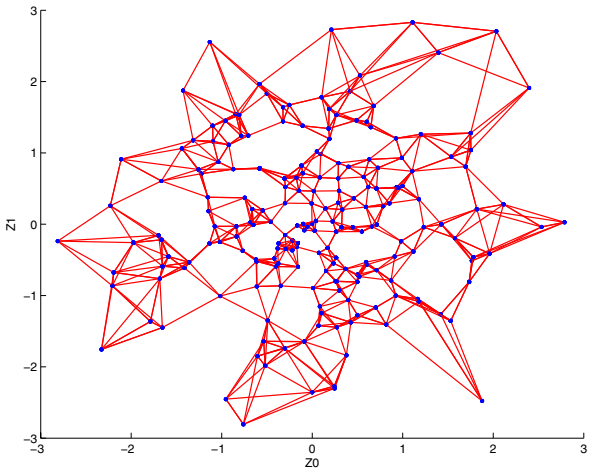

(b)

Fig. 1. $\mathrm{kNN}$ graphs for a set of 200 points in the plane and $\mathrm{k}=5$. (a) Uniform distribution $(\mathrm{SD}=1)$. (b) Gaussian distribution $(\mathrm{SD}=1)$.

$$
\begin{aligned}
\alpha M I & =D_{\alpha}\left(p_{s t}\left(I_{s}, I_{t}\right) \| p_{s}\left(I_{s}\right) p_{t}\left(I_{t}\right)\right) \\
& =\frac{1}{\alpha-1} \log \int p_{s t}^{\alpha}\left(I_{s}, I_{t}\right) p_{s}^{1-\alpha}\left(I_{s}\right) p_{t}^{1-\alpha}\left(I_{t}\right) d I_{s} d I_{t} .
\end{aligned}
$$

When $\alpha \rightarrow 1, \alpha \mathrm{MI}$ converges to the standard (Shannon) MI

$$
M I=\int p_{s t}\left(I_{s}, I_{t}\right) \log \left(\frac{p_{s t}\left(I_{s}, I_{t}\right)}{p_{s}\left(I_{s}\right) p_{t}\left(I_{t}\right)}\right) d I_{s} d I_{t} .
$$

According to Equation (2), $\alpha \mathrm{MI}$ can be interpreted as a measure of dependency between variables $I_{s}$ and $I_{t}$, which is expected to be maximum at registration.

\section{$2.3 \alpha$ MI Estimation}

Given a set $\mathcal{Z}=\left\{z_{1}, \ldots, z_{n}\right\}$ of $n$ vectors in $\mathbb{R}^{d}$, the k-Nearest Neighbor Graph (kNN Graph) is formed by the points $z_{i}$ and the edges with their $k$ nearest points $\mathcal{N}_{k, i}(\mathcal{Z})$. This graph belongs to a particular class of graphs known as Entropic Spanning Graphs (ESGs), whose relationship to alpha entropy is described in 14. Figure 1 shows two examples of $\mathrm{kNN}$ graphs for different distributions.

Let $I_{s}$ and $I_{t}$ be two images from which the sets of feature vectors $\mathcal{Z}_{s}=$ $\left\{z_{s 1}, \ldots, z_{s n}\right\}$ and $\mathcal{Z}_{t}=\left\{z_{t 1}, \ldots, z_{t n}\right\}$ have been extracted. kNN graphs allow for estimating $\alpha \mathrm{MI}$ between these images as [16]

$$
\widehat{\alpha M I}=\frac{1}{\alpha-1} \log \frac{1}{n^{\alpha}} \sum_{i=1}^{n} \sum_{p=1}^{k}\left(\frac{\left\|e_{i p}\left(z_{s i}, z_{t i}\right)\right\|}{\sqrt{\left\|e_{i p}\left(z_{s i}\right)\right\|\left\|e_{i p}\left(z_{t i}\right)\right\|}}\right)^{2 \gamma},
$$

where $\left\|e_{i p}\left(z_{s i}, z_{t i}\right)\right\|$ is the distance from the point $\left(z_{s i}, z_{t i}\right) \in \mathbb{R}^{2 d}$ to its $p$-nearest neighbor in $\left\{z_{s j}, z_{t j}\right\}_{j \neq i}$, and $\left\|e_{i p}\left(z_{s i}\right)\right\|\left(\left\|e_{i p}\left(z_{t i}\right)\right\|\right)$ is the distance from the point $z_{s i} \in \mathbb{R}^{d},\left(z_{t i} \in \mathbb{R}^{d}\right)$ to its $p$-nearest neighbor in $\left\{z_{s j}\right\}_{j \neq i}\left(\left\{z_{t j}\right\}_{j \neq i}\right)$.

In this work we used $\alpha=0.5, \gamma=2, n \simeq 1000$ (it depends on the LV area), $k=4$ and $\epsilon=0.1$, the maximum allowed error between a point and its nearest neighbor. 


\subsection{Feature Vectors}

There are many possible feature vectors (FV) to be used in $\alpha \mathrm{MI}$ based techniques. In this work, in order to obtain the feature vector corresponding to the point $\mathbf{x}_{0}$, we applied the Discrete Wavelet Transform (DWT) to decompose the image $I(\mathbf{x})$ into four subimages $I_{L L}(\mathbf{x}), I_{L H}(\mathbf{x}), I_{H L}(\mathbf{x})$ and $I_{H H}(\mathbf{x})$. Then, we defined the $\mathrm{FV}$ of point $\mathbf{x}_{0}$ by taking the corresponding wavelet coefficients as:

$$
\mathbf{z}=\left[I_{L L}(\mathbf{x}) I_{L H}(\mathbf{x}) I_{H L}(\mathbf{x}) I_{H H}(\mathbf{x})\right]
$$

This paper is not focused on finding the optimal feature for this particular application, but on evaluating the effect of introducing spatial information into the objective function. Therefore, for a first approach, we chose Haar wavelet coefficients owing to its well known ability for edge detection and simplicity. This basis was expected to perform well defining tags in MRI images and thus good for guiding the registration process.

\section{Materials}

\subsection{Dataset}

Two tagged 2D sequences were acquired with a GE Genesis Signa 1.5T MRI scanner. A cine breath-hold sequence with a SPAMM grid tag pattern was used, with imaging being done at end expiration. The in-plane image resolution was $1.56 \mathrm{~mm} \times 1.56 \mathrm{~mm}$. Cardiac cycle was sampled by acquiring a total of 16 frames. However, only images from End of Diastole (ED) to End of Systole (ES) (systolic phase) were used in the experiments due to our interest on evaluating deformation during heart contraction. The length of this cardiac cycle segment is 5 frames.

\subsection{Manual Measurements}

In order to assess the method performance in tracking myocardial motion, tagintersection points were marked manually in each frame by two observers in two independent sessions. For each sequence, 22 points were chosen to be tracked, and

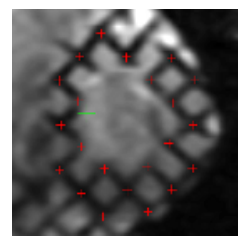

(1)

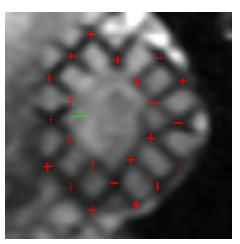

(2)

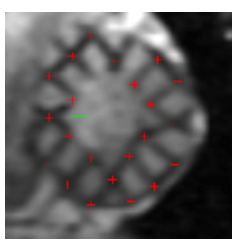

(3)

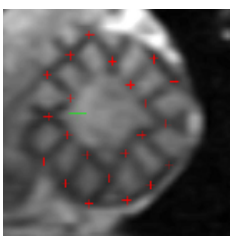

(4)

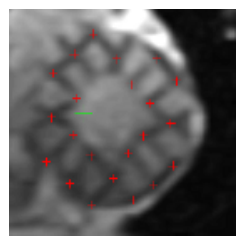

(5)

Fig. 2. Gold standard point positions in each frame from ED to ES for one of the sequences used in this work 
thus $110(22 \times 5)$ points were marked. Gold-standard measurements were derived for each tag-intersection point by taking the average of the measurements made by the observers. Figure 2 shows the gold-standard landmarks for each frame in sequence A.

\section{Results}

The mean error between the gold standard points and the corresponding positions assessed by the observers was calculated. Table 1 shows the intra and interobserver variabilities of manual landmarking.

The deformation field of the myocardium was calculated with the method explained in Section 2 The resulting transformations were then applied to the gold standard pointset at ED to map these points to each phase. The mean error between these mapped points and the actual positions according to the gold standard was calculated. Figure 3 shows this error from ED to ES. With both methods, subpixel accuracy was obtained for all the phases in patient A and for the two first phases in patient B. Figure 4 shows the initial frames of both sequences, along with an arrow plot showing the displacement field in the myocardium during systole.

Table 1. Accuracy of manual measurements. Bias and standard deviation of the differences, corrected for repeated measurements, between manual and gold standard measurements

\begin{tabular}{|c|c|c|c|}
\hline & Observer $A$ & Observer $B$ & Observer $A$ and $B$ \\
\hline \hline Bias $(\mathrm{mm})$ & 0.01 & 0.06 & 0.03 \\
\hline SD $(\mathrm{mm})$ & 0.35 & 0.31 & 0.29 \\
\hline
\end{tabular}

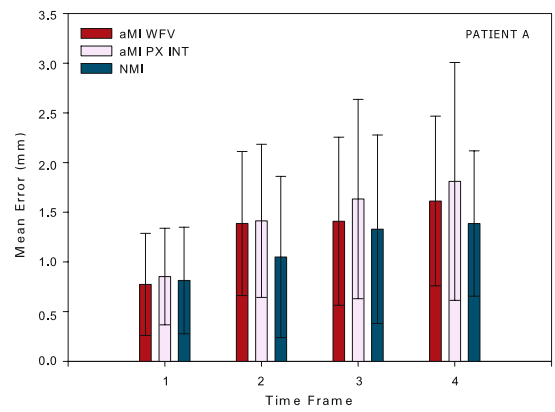

(a)

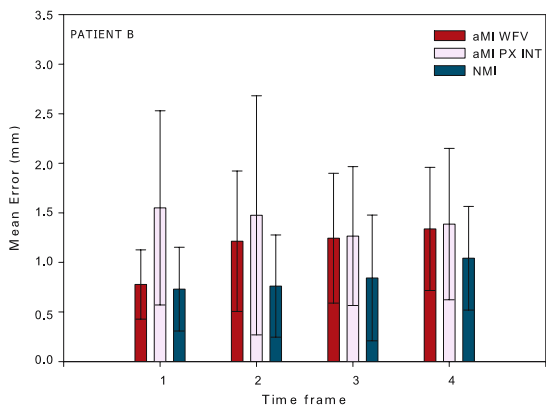

(b)

Fig. 3. Mean error in landmark correspondence between the gold-standard position and the position of the landmarks in end-diastole after being transformed through the computed deformation field. Results for the different registration metrics is provided. (a) Patient A. (b) Patient B. 


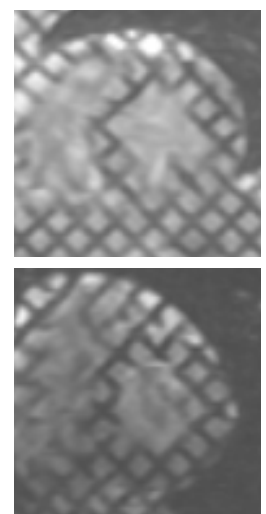

(a)

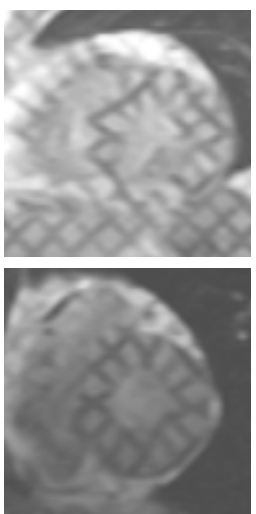

(b)

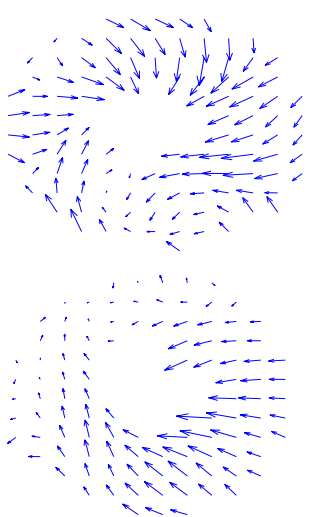

(c)

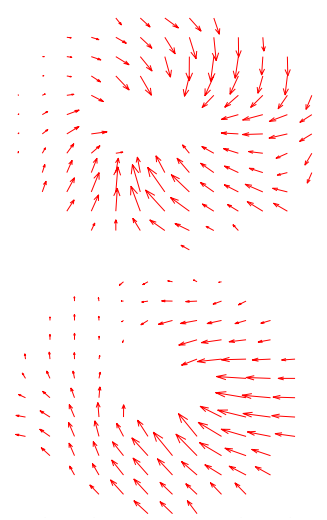

$(d)$

Fig. 4. Displacement of myocardium points during heart contraction by using non-rigid registration for sequence A (top row) and sequence B (bottom row). (a) ED frame. (b) ES frame. (c) Motion field close-up obtained by using $\alpha \mathrm{MI}$. (d) Motion field close-up obtained by using NMI.

\section{Discussion}

In this paper we have applied an ESG estimation of $\alpha \mathrm{MI}$ for myocardial motion estimation. The results show low mean error values with respect to the gold standard measurements, which demonstrate that this method allows retrieving cardiac motion fields accurately.

For this particular application, and according to Figure 3, the use of NMI seems to give better results. However, the standard deviations of the errors are high, and therefore these differences are statistically not significant. A possible explanation for these differences may derive from the feature definition. In this work we have used Haar wavelet coefficients because of their well known ability for edge detection, which was expected to perform appropriately in detecting tag borders. However, Haar basis presents a lack of invariance to translation and rotation which can be corrected by using "cycle spanning" or complex wavelets. Haar basis also has an inherent lack of sensitivity to edge deformations which dominate the deformation feature space. Regarding this matter, a smoother basis like Daubechies or Curvelets might have better potential.

Another explanation for the differences with respect to NMI, is that estimating $\alpha \mathrm{MI}$ in multidimensional spaces may introduce more local minima in the error surface than conventional NMI. Thus, the lower accuracy may arise as a consequence of using a local optimization like the downhill method used in this work. Finally, the image resolution of MRI may justify some of the disagreement between measurements. ESGs allow to estimate $\alpha \mathrm{MI}$ accurately when the number of FVs used to calculate the graph is large. Given that the in-plane resolution is $1.56 \mathrm{~mm} \times 1.56 \mathrm{~mm}$ and that only the $\mathrm{LV}$ was considered, less than 1000 points were available for $\alpha \mathrm{MI}$ estimation. 
Figure 4 shows a good agreement between estimated displacements fields of each metric for sequence B. For sequence A, there was clearly a different result in the lower-right portion of the image. The frame at ES for this patient shows completely vanished tags and poor image quality in the part of the myocardium where the motion field is altered. Therefore, it could be hypothesized that the incorporation of spatial features into the objective function makes the results more dependent on the presence of such features in the target and source images.

With respect to the extension of this work to the three dimensional case, the main drawback is that ESGs are computationally expensive. However, many algorithms have been developed to compute graphs in an approximated manner, allowing a significant speed up of the graph construction.

\section{Conclusions}

Entropic spanning graph estimation of $\alpha \mathrm{MI}$ has been applied for non rigid registration for the first time and has proven to retrieve myocardial deformation fields accurately. Although the results were quite satisfactory, even lower errors have been obtained with NMI. However, the observed differences were statistically not significant and further research needs to be done to fully understand the reason of this behavior. ESGs offer an increased flexibility in the kinds of features one can use for these types of problems, and further research needs to be done regarding this matter.

\section{Acknowledgements}

This work was partially funded by Red IM3: Imagen Mdica Molecular y Multimodalidad (ISCIII G03/185), Redes Temticas Colaborativas, Ministerio de Sanidad y Consumo de Espaa (http://im3.rediris.es) and TIC2002-04495-C02 grant. The work of E. Oubel is supported by the Spanish Ministry of Education under a FPU Grant AP2003-1535. The work of A.F. Frangi is supported by the Spanish Ministry of Science and Technology under a Ramn y Cajal Research Fellowship. Special acknowledgements to Dra. Marina Huguet from Centro Cardiovascular Sant Jordi, Barcelona, Spain, for providing the images used in the experiments and to GridSystems S.A., Palma de Mallorca, Spain (http://www.gridsystems.com) for providing the InnerGrid Nitya Middleware for grid computing and technical support.

\section{References}

1. Axel, L., Dougherty, L.: MR imaging of motion with spatial modulation of magnetization. Radiology 171 (1989) 841-845

2. Zerhouni, E., Parish, D., Rogers, W.: Human heart: tagging with MR imaging - a method for non invasive assessment of myocardial motion. Radiology 169 (1988) $59-63$ 
3. Prince, J., McVeigh, E.: Motion estimation from tagged MR image sequences. IEEE Trans. Med. Imag. 11 (1992) 238-249

4. Gupta, S., Prince, J.: On variable brightness optical flow for tagged MRI. In: Inform. Processing Med. Imag. (1995) 323-334

5. Osman, N., Kerwin, W., McVeigh, E., Prince, J.: Cardiac motion tracking using cine harmonic phase (HARP) magnetic resonance imaging. Magn. Reson. Med. 42 (1999) 1048-1060

6. Osman, N., McVeigh, E., Prince, J.: Imaging heart motion using harmonic phase MRI. IEEE Trans. Med. Imag. 19 (2000) 186-202

7. Kumar, S., Goldgof, D.: Automatic tracking of SPAMM grid and the estimation of deformation parameters from cardiac MR images. IEEE Trans. Med. Imag. 13 (1994) 122-132

8. Guttman, M., Prince, J., McVeigh, E.: Tag and contour detection in tagged MR images of the left ventricle. IEEE Trans. Med. Imag. 13 (1994) 74-88

9. Kraitchman, D., Young, A., Change, C., Axel, L.: Semi-automatic tracking of myocardial motion in MR tagged images. IEEE Trans. Med. Imag. 14 (1995) 422-433

10. Clarysse, P., Basset, C., Khouas, L., Croisille, P., Friboulet, D.: Two-dimensional spatial and temporal displacement and deformation field fitting from cardiac magnetic resonance imaging. Medical Image analysis 4 (2000) 253-268

11. Petitjean, C., Rougon, N., Prêteux, F., Cluzel, P., Grenier, P.: Measuring myocardial deformations from MR data using information-theoretic non rigid registration. In: Proceedings Second International Workshop on Functional Imaging and Modeling of the Heart (FIMH'2003), Lyon, France - Lecture Notes in Computer Science 2674, Heidelberg, Germany, Springer Verlag (2003) 162-172

12. Chandrasheckara, R., Mohiaddin, R.H., Rueckert, D.: Analysis of 3D myocardial motion in tagged MR images using non rigid image registration. IEEE Trans. Med. Imag. 23 (2004) 1245-1250

13. Rueckert, D., Sonoda, L.I., Hayes, C., Hill, D.L.G., Leach, M.O., Hawkes, D.J.: Nonrigid registration using free-form deformations: application to breast MR images. IEEE Trans. Med. Imag. 18 (1999) 712-721

14. Hero, A.O., Ma, B., Michael, O., Gorman, J.: Applications of entropic spanning graphs. IEEE Signal Processing Magazine (Special Issue on Mathematics in Imaging) 19 (2002) 85-95

15. Schnabel, J.A., Rueckert, D., Quist, M., Blackall, J.M., Castellano-Smith, A.D., Hartkens, T., Penney, G.P., Hall, W.A., Liu, H., Truwit, C.L., Gerritsen, F.A., Hill, D.L.G., Hawkes, D.J.: A generic framework for nonrigid registration based on non uniform multi-level free-form deformations. In Niessen, W.J., Viergever, M.A., eds.: Proc. 4th Int. Conf. Medical Image Computing and Computer Assisted Intervention, Utrecht, The Netherlands (2001) 573-581

16. Neemuchwala, H.F., Hero, A.O.: Entropic graphs for registration. In: Multi-sensor image fusion and its applications. Marcel-Dekker, Inc. (2004) 